\title{
Corrigendum: SAMHD1 is a biomarker for cytarabine response and a therapeutic target in acute myeloid leukemia
}

Constanze Schneider, Thomas Oellerich, Hanna-Mari Baldauf, Sarah-Marie Schwarz, Dominique Thomas, Robert Flick, Hanibal Bohnenberger, Lars Kaderali, Lena Stegmann, Anjali Cremer, Margarethe Martin, Julian Lohmeyer, Martin Michaelis, Veit Hornung, Christoph Schliemann, Wolfgang E Berdel, Wolfgang Hartmann, Eva Wardelmann, Federico Comoglio, Martin-Leo Hansmann, Alexander F Yakunin, Gerd Geisslinger, Philipp Ströbel, Nerea Ferreirós, Hubert Serve, Oliver T Keppler \& Jindrich Cinatl Jr

Nat. Med. 23, 250-255 (2017); published online 19 December 2016; corrected after print 18 April 2017

In the version of this article initially published, the Acknowledgements section contained an error: "The WEBs laboratory is supported by the Deutsche Forschungsgemeinschaft grant DFG EXC 1003 (H.S.)." is incorrect. The correct sentence is: "W.E.B.s laboratory is supported by the Deutsche Forschungsgemeinschaft grant DFG EXC 1003.” The error has been corrected in the HTML and PDF versions of the article.

\section{Erratum: PIM1 kinase regulates cell death, tumor growth and chemotherapy response in triple-negative breast cancer}

Fara Brasó-Maristany, Simone Filosto, Steven Catchpole, Rebecca Marlow, Jelmar Quist, Erika Francesch-Domenech, Darren A Plumb, Leila Zakka, Patrycja Gazinska, Gianmaria Liccardi, Pascal Meier, Albert Gris-Oliver, Maggie Chon U Cheang, Anna Perdrix-Rosell, Manar Shafat, Elodie Noël, Nirmesh Patel, Kristen McEachern, Maurizio Scaltriti, Pau Castel, Farzana Noor, Richard Buus, Sumi Mathew, Johnathan Watkins, Violeta Serra, Pierfrancesco Marra, Anita Grigoriadis \& Andrew N Tutt

Nat. Med. 22, 1303-1313 (2016); published online 24 October 2016; corrected after print 26 January 2017

In the version of this article initially published, the western blot in Figure 2a for the BT474 cell line was incorrect. In addition, one of the accession codes for gene expression data for the Guy's Hospital TNBC-enriched cohort was incorrect. The errors have been corrected in the HTML and PDF versions of the article.

\section{Erratum: Blocking microglial pannexin-1 channels alleviates morphine withdrawal in rodents}

\author{
Nicole E Burma, Robert P Bonin, Heather Leduc-Pessah, Corey Baimel, Zoe F Cairncross, Michael Mousseau, Jhenkruthi Vijaya Shankara, \\ Patrick L Stemkowski, Dinara Baimoukhametova, Jaideep S Bains, Michael C Antle, Gerald W Zamponi, Catherine M Cahill, \\ Stephanie L Borgland, Yves DeKoninck \& Tuan Trang \\ Nat. Med.; doi:10.1038/nm.4281; corrected online 15 February 2017
}

In the version of this article initially published online, Yves De Koninck's name was misspelled in the author list. The original version listed Yves DeKoninck. The error has been corrected in the print, PDF and HTML versions of this article.

\section{Erratum: Oncostatin M drives intestinal inflammation and predicts response to tumor necrosis factor-neutralizing therapy in patients with inflammatory bowel disease}

\footnotetext{
Nathaniel R West, Ahmed N Hegazy, Benjamin M J Owens, Samuel J Bullers, Bryan Linggi, Sofia Buonocore, Margherita Coccia, Dieter Görtz, Sébastien This, Krista Stockenhuber, Johanna Pott, Matthias Friedrich, Grigory Ryzhakov, Frédéric Baribaud, Carrie Brodmerkel, Constanze Cieluch, Nahid Rahman, Gerhard Müller-Newen, Raymond J Owens, Anja A Kühl, Kevin J Maloy, Scott E Plevy, Oxford IBD Cohort Investigators, Satish Keshav, Simon P L Travis \& Fiona Powrie Nat. Med.; doi:10.1038/nm.4307; corrected online 11 April 2017
}

In the version of this article initially published, there were two typographical errors in the Abstract. The unnecessary ' $h$ ' in the line "Furthermore, $\mathrm{h}$ according to....." has been deleted. The line "OSM is thus a potential biomarker of and therapeutic target for IBD,...." was changed to read "OSM is thus a potential biomarker and therapeutic target for IBD...”. These errors have been corrected in the HTML and PDF versions of the article. A coding error that inadvertently resulted in incorrect ordering of the authors in the HTML version was also corrected. 